\title{
Behaviour fuels, and fights, pandemics
}

\author{
Insight into human behaviour is key to understanding both the systemic causes of the COVID-19 pandemic and \\ how we can act to mitigate its impacts. Both now and in its wake, we have the capacity to shape and reshape the
} world we live in.

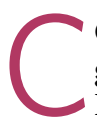
OVID-19 escalated from outbreak to global pandemic in a matter of weeks. In the absence of pharmaceutical routes to curb the virus, human behaviour has the power to both facilitate and contain its transmission and consequences. This has cast contemporary human behaviour into the spotlight: individual, group and political thought and action are under new scrutiny. The Nature Human Behaviour community spans the broad reach of the social and behavioural sciences, and in this Focus Issue we feature opinion and comment contributed by experts from a wide range of disciplines. In the coming weeks and months we will be expanding this as a Collection, to which we will add new research and non-research content on COVID-19 published in the journal.

Notably, all authors in this Focus emphasise the importance of perspective, which we too feel is crucial at this time. Critique, analysis and reflection have an important role to play. In addition, even though many are locked down in the very thick of a global crisis, our actions today hold a great deal of power. With every second that passes, the number of cases rises worldwide. This urgent timeline sends a powerful message: our behaviour aids, but crucially can also forestall, the spread of the virus. We need to fight in real time, using the capacity we each have to curb transmission.

As we do so, it is tempting to imagine a future in which we emerge into a
post-COVID world with a collective sigh of relief. It will differ from the normality we once recognised. While we muse about its landscape, we must also bear in mind that very little about it is inevitable. We include in this issue a Research Highlight covering a paper published in 1919 after the world's last great pandemic event, which shows that while much has changed since 1919, much remains the same (https://doi.org/10.1038/ s41562-020-0876-z). Importantly, we now have the opportunity to learn from the past in the wake of disaster.

In recent months, we have seen rapid changes in welfare provisions, public health spending and scientific publishing. The latter has moved fast in response to COVID-19, with immediate free access to content, mandatory preprint deposition and rapid peer review all becoming the new normal for many journals. Crisis measures are often rolled out with the acknowledgement that they may be unsustainable ways in which we fight in the moment. Yet their efficacy can also inform how we behave in the aftermath.

In the midst of this pandemic, there is a danger that human behaviour is also perpetuating and exacerbating patterns that have long had devastating consequences: racism, xenophobia, restrictions on personal freedom, and extreme inequality. The rise in Asian-directed racist attacks worldwide (https://www.nature.com/ articles/d41586-020-01009-0) and the disproportionate number of Black and minority ethnicity (BME) patients critically ill with COVID-19 in UK hospitals (https://www.aljazeera.com/news/2020/04/ critically-ill-covid-19-uk-patients-bme-back grounds-200407143303604.html) are just two such examples. The idea that the virus does not see class is a dangerous myth: the biological entity may not, but its consequences certainly do. The privileged can access testing, high quality care and state benefits, while the poor, homeless and displaced are less fortunate. The disadvantaged have become even more disadvantaged, and we must not lose sight of this stark reality as we fight to mitigate the global health and economic consequences of COVID-19.

In the face of a global pandemic, we all feel powerless at times. Scientists, journals, funders and academic institutions all have a role to play. We acknowledge that inequality is also rife in our own communities, and this too must be attenuated by us all.

The encouraging message of social and behavioural science is that every one of us has some capacity to act, both in the short term and the longer term: as scientists, as editors, as policymakers, and also as colleagues, as friends and as family. As a journal we hope to play our part in curbing this virus and, cautiously, in carving a post-COVID future.

Published online: 19 May 2020

https://doi.org/10.1038/s41562-020-0892-z 